\title{
Flagellin shifts 3D bronchospheres towards mucus hyperproduction
}

\author{
Richard F. Sprott ${ }^{1 \dagger}$, Felix Ritzmann ${ }^{1 \dagger}$, Frank Langer ${ }^{2}$, Yiwen Yao ${ }^{1}$, Christian Herr ${ }^{1}$, Yvonne Kohl ${ }^{3}$, Thomas Tschernig ${ }^{4}$, \\ Robert Bals ${ }^{1+}$ and Christoph Beisswenger ${ }^{1 * \dagger}$ (i)
}

\begin{abstract}
Cystic fibrosis (CF) and chronic obstructive pulmonary disease (COPD) are associated with acute and chronic bacterial infections of the lung. Excessive differentiation of basal cells to mucus-producing goblet cells can result in mucus hyperproduction and loss of mucociliary clearance in the airways of CF and COPD patients. Here, we aimed to investigate the effect of pathogen-associated molecular patterns (PAMPs) on the differentiation of human 3D bronchospheres. Primary human bronchial epithelial cells (HBECs) were differentiated to bronchospheres in the presence of bacterial flagellin and LPS and the synthetic Toll-like receptor (TLR) ligands Pam3CSK4 (TLR-2) and polyinosinic:polycytidylic acid (pIC, TLR-3). Electron and fluorescence microscopy showed that the differentiation of bronchospheres associated with the formation of lumina and appearance of cilia within 30 days after seeding. Incubation with flagellin resulted in a decreased formation of lumina and loss of cilia formation. Incubation with Pam3CSK, plC, and LPS did not significantly affect formation of lumina and ciliation. Mucus production was strongly increased in response to flagellin and, to a lesser degree, in response to Pam3CSK4. Our results indicate that bacterial factors, such as flagellin, drive the differentiation of the respiratory epithelium towards mucus hyperproduction.
\end{abstract}

Keywords: Organoids, Bronchospheres, Mucus, PAMPs, Flagellin

\section{Introduction}

Airway remodeling contributes to air flow limitation and loss of lung structure in chronic respiratory diseases, such as CF and COPD. In both diseases, basal and goblet cell hyperplasia leads to mucus hyperproduction, loss of mucociliary clearance, disturbed epithelial barrier function, and impaired regeneration of the airway epithelium $[1,2]$. Therefore, the respiratory epithelium is a potential target for therapeutic intervention addressing basal cell differentiation and mucus hyperproduction [1].

\footnotetext{
* Correspondence: Christoph.Beisswenger@uks.eu

${ }^{\dagger}$ Richard F. Sprott, Felix Ritzmann, Robert Bals and Christoph Beisswenger contributed equally to this work.

${ }^{1}$ Department of Internal Medicine V - Pulmonology, Allergology and Respiratory Critical Care Medicine, Saarland University, Kirrberger Str. 100, Building 41M, 66421 Homburg/Saar, Germany

Full list of author information is available at the end of the article
}

CF patients suffer from persistent bacterial infections of the lung with Pseudomonas aeruginosa being a dominant pathogen later in life [3]. In COPD, nontypeable Haemophilus influenzae (NTHi) contributes to pulmonary inflammation and acute exacerbations [4]. Infections and colonization in the lung with these pathogens associate with a constant contact of the airway epithelium with PAMPs (e.g. lipopeptide, LPS, flagellin) which activate innate immunity responses via pattern recognition receptors (PRRs), such as TLRs. Even though the initiation of innate immunity is required for an adequate host response, prolonged and excessive activation of innate inflammatory responses may be harmful and therefore contribute to the progression of CF and COPD [3-6].

In recent years, 3D organoid models have been established to study tissue differentiation and therapeutic interventions. Pre-clinical studies suggest that transplanted 
primary lung organoids can be used for cell-based therapies for the treatment of chronic lung diseases, such as COPD or IPF [7-9]. Importantly, 3D organoid models have the potential to reduce and replace animal studies. A recent manuscript showed that exposure of developing bronchospheres to inflammatory mediators (e.g. IL-13) drives goblet cell metaplasia [10]. The purpose of this study was to investigate the consequences of the exposure to PAMPs on the differentiation of human 3D bronchospheres. We demonstrate that bacterial-derived factors, such as flagellin, shift 3D bronchospheres towards mucus hyperproduction. Moreover, exposure to flagellin resulted in a loss of ciliation.

\section{Methods}

HBECs were isolated from patients who underwent surgery because of lung cancer as described previously [11]. Only cancer-free tissue was used for cell isolation. The protocol was approved by the institutional review board (ethics committee) of the Landesärztekammer of the State of Saarland, and informed consent was obtained from the patients. Organoids were cultured as described before [10]. In brief, $80 \mu \mathrm{l}$ of passage 1 cells $\left(3 \times 10^{4}\right.$ cells per $\mathrm{ml}$ differentiation media containing $5 \%$ Matrigel) were plated in each well of a 96-well plate containing $40 \mu \mathrm{l}$ per well of a $25 \%$ Matrigel (Corning, USA) solution as base layer. $120 \mu \mathrm{l}$ differentiation media was added at day 2 containing $100 \mathrm{ng} / \mathrm{ml}$ of Pam3CSK4, polyinosinic: polycytidylic acid (pIC), ultrapure LPS from Escherichia coli 055:B5, or flagellin from $P$. aeruginosa (Invivogen, USA) and 5\% Matrigel. Media was changed every 4 days. The presence of lumina, cilia beating, and diameter were determined under the phase contrast microscope 14, 22, and 30 days after seeding. Organoids from each condition were pooled and embedded in paraffin 30 days after seeding. Paraffin sections ( $2 \mu \mathrm{M}$ thickness) were stained with haematoxilin-eosin (H\&E). Deparaffinized paraffin sections were treated with BSA (1\%) and Tween-20 in PBS $(0.1 \%)$ and incubated overnight at $4{ }^{\circ} \mathrm{C}$ with the primary antibodies for MUC5B (sc-393,952, Santa Cruz, USA, 1/100) and KRT5 (ab-75,869, Abcam, USA, 1/100) in PBS containing BSA (1\%). Cells were incubated for $30 \mathrm{~min}$ with secondary antibodies (goat anti-mouse FITC, Sigma, Germany; goat anti-rabbit, Cyanine 5, Thermo-Fisher Scientific, Germany). Immunohistochemistry for MUC5AC (ab-3649, Abcam) was performed as described before [12]. To analyze and merge images, we used ImageJ software (National Institutes of Health). Western blot was performed as described before [12]. Membranes were probed with monoclonal antibodies directed against MUC5B (sc-393,952, Santa Cruz) and $\beta$-actin (\#4967, Cell Signaling Technology, USA). The ultrastructure of untreated bronchospheres were analyzed by electron microscopy as described before [13]. Ultra-thin sections were contrasted with uranylacetate using routine procedures. The analysis was performed using a FEI Technai 12 transmission electron microscope (FEI, Thermo-Fisher Scientific) at $100 \mathrm{kV}$, equipped with a digital 8-bit camera. Comparisons between groups were analyzed by $\mathrm{t}$ test (two-sided) using the software Prism (GraphPad Software, USA). Technical replicates were combined to a single data point for each independent experiment. The results were considered statistically significant for $P<0.05$.

\section{Results}

Transmission electron microscopy and H\&E staining showed that HBECs seeded in matrigel formed differentiated bronchospheres with microvilli and typical kinocilia on its surface (Fig. 1a and b). We studied whether PAMPs modulate the differentiation of bronchospheres. Therefore, we treated the developing bronchospheres with different ligands for TLRs (Pam3CSK4, pIC, LPS, flagellin) during the differentiation phase starting at day 2 after seeding. Examination under the phase contrast microscope showed that the differentiation of bronchospheres associated with the formation of lumina preceding visible cilia beating within 30 days after seeding (Fig. 1c to e). Treatment with flagellin resulted in an almost complete loss of visible cilia beating, but did not significantly affect formation of lumina and the diameter of the bronchospheres. Treatment with Pam3CSK4, pIC, and LPS did not significantly affect formation of lumina and ciliation. H\&E staining of bronchospheres embedded 30 days after seeding confirmed that treatment with flagellin but not Pam3CSK4, pIC, and LPS inhibited the formation of cilia (Fig. 1f). Ciliated cells were identified in approximately $75 \%$ of control bronchospheres, whereas no ciliated cells were found in bronchospheres treated with flagellin (Fig. 1g). Moreover, the percentage of organoids with a lumen was significantly reduced in the flagellin-treated group (Fig. 1h). H\&E staining of paraffin embedded bronchospheres confirmed the presence of cilia in control bronchospheres, but not in the flagellin treated group 21 days after seeding (Fig. 1i).

To study whether the different conditions affected mucus production we stained bronchospheres for the goblet cell marker mucin 5B (MUC5B) and cytokeratin 5 (KRT5), a marker for basal cells, 30 days after seeding (Fig. 2a). Quantification of the staining showed that treatment with flagellin resulted in a strongly increased expression of MUC5B (Fig. 2b), whereas there was no significant difference in the expression of KRT5 (Fig. 2c). Treatment with Pam3CSK also resulted in a significantly increased expression of MUC5B. In addition, western blot analysis demonstrated an increased 
A

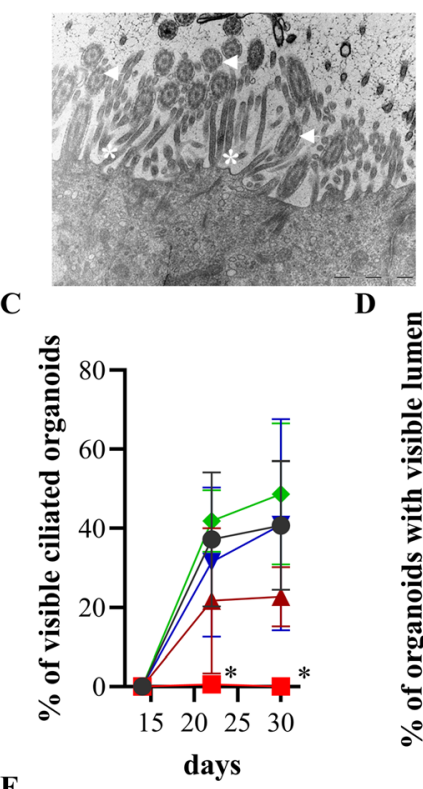

F
B
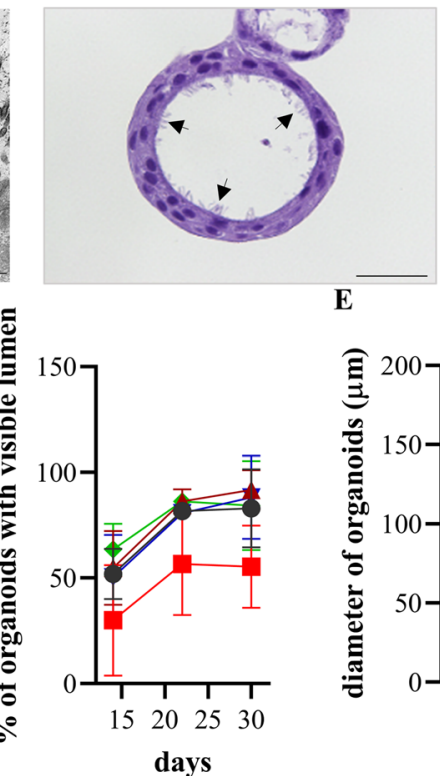

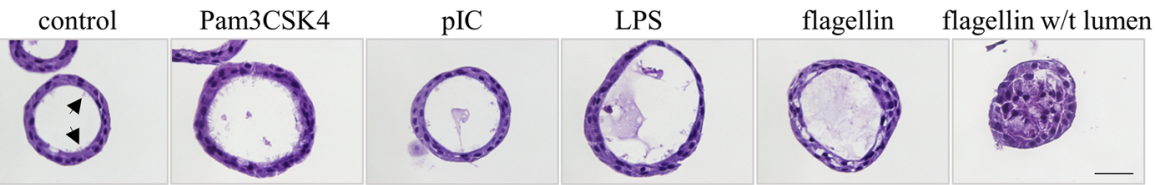

G

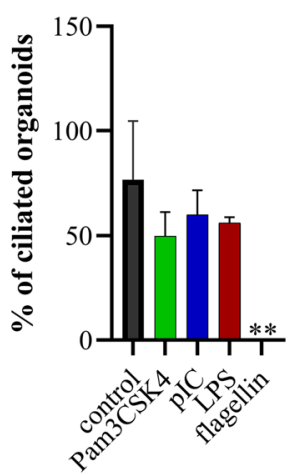

$\mathbf{H}$

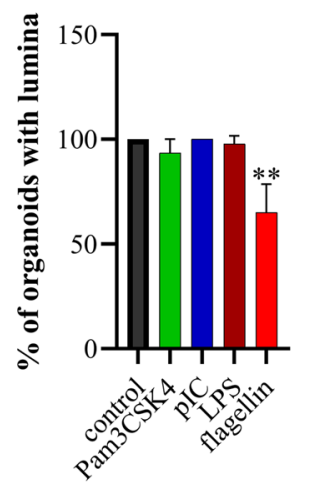

I

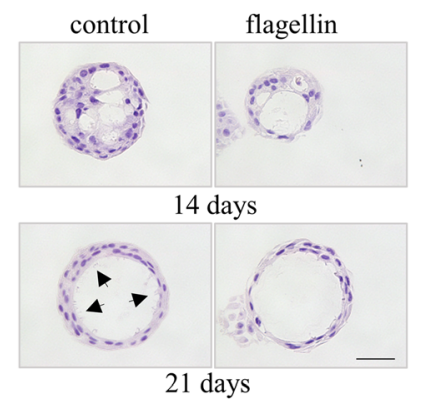

Fig. 1 Flagellin inhibits the formation of cilia. a The ultrastructure of the inner surface of a bronchosphere embedded 30 days after seeding (23.000-fold magnification). Arrows indicate kinocilia and * indicate microvilli. b Representative H\&E staining of a bronchosphere embedded 30 days after seeding. Scale bar: $20 \mu \mathrm{m}$. Arrows indicate cilia. (c to f) Developing bronchospheres were treated with different PAMPs during the differentiation phase starting at day 2 after seeding. Cilia beating (c), the presence of lumina (d), and diameter (e) were determined under the phase contrast microscope 14,22, and 30 days after seeding. (f) Representative H\&E staining of bronchosphere cultured under the indicated conditions embedded 30 days after seeding. Arrows indicate cilia. Scale bar: $20 \mu \mathrm{m}$. The percentage of bronchospheres with cilia (g) and lumina (h) were analyzed H\&E-stained sections. Data were compared by unpaired Student's t-test and are shown as the mean \pm SEM from three independent experiments. ${ }^{*} p<0.05,{ }^{*} p<0.01$. (i) H\&E staining of bronchospheres cultured under the indicated conditions embedded 14 and 21 days after seeding. Arrows indicate cilia. Scale bar: $20 \mu \mathrm{m}$

expression of MUC5B in flagellin-treated bronchospheres 30 days after seeding of the basal cells (Fig. 2d). Immunohistochemistry further showed that treatment with flagellin also resulted in an increased expression of the mucin MUC5AC (Fig. 2 e and f).

\section{Discussion}

CF and COPD are characterized by chronic bacterial colonization of the airways $[3,4]$. Therefore, the airway epithelium of CF and COPD patients is constantly exposed to bacterial components (e.g. flagellin, LPS, 


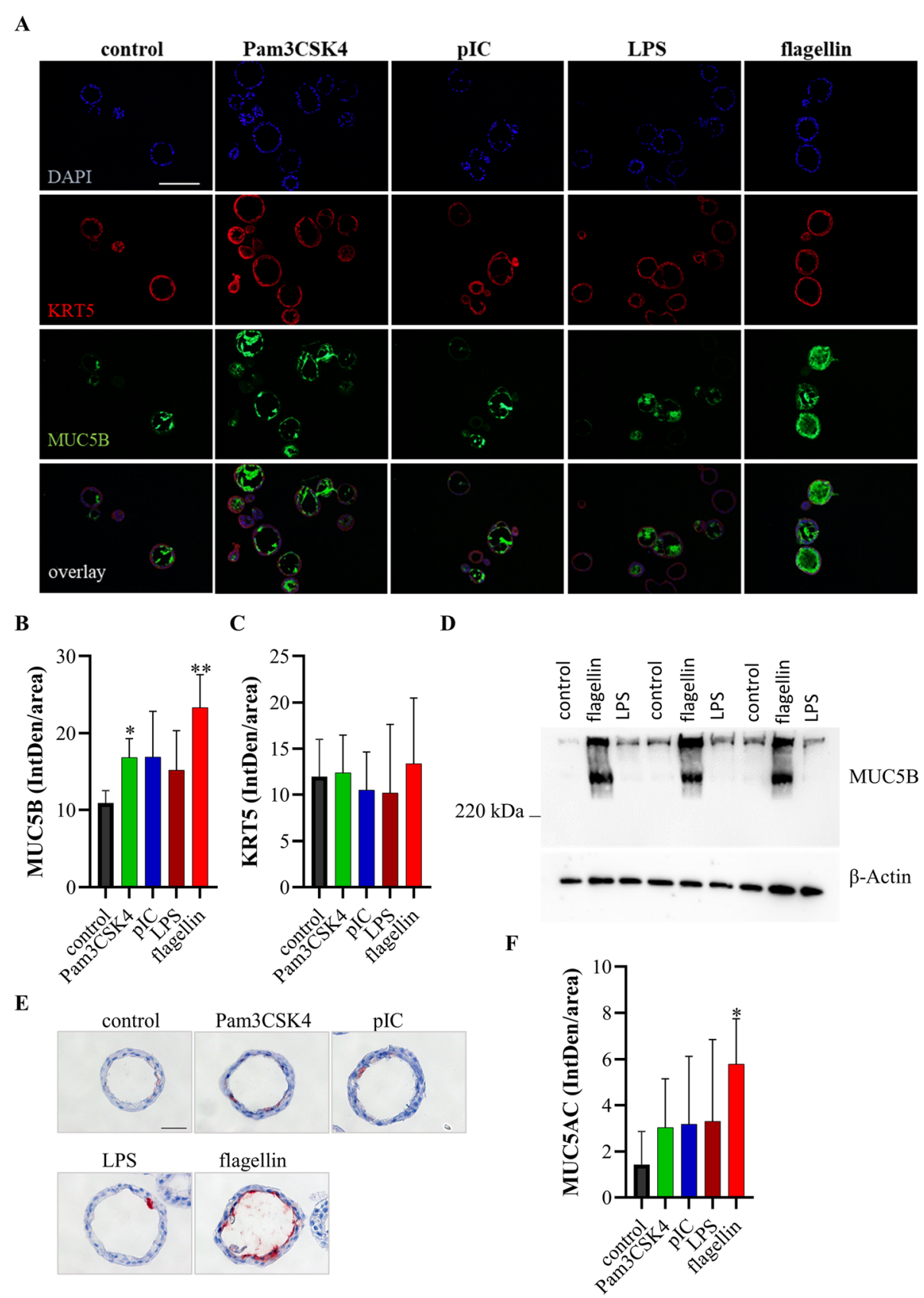

Fig. 2 Flagellin induces mucus secretion. Developing bronchospheres were treated with different PAMPs during the differentiation phase starting at day 2 after seeding. Bronchospheres were analyzed 30 days after seeding. a Immunofluorescence staining was performed for KRT5 (red channel) and MUC5B (green channel). Nuclei were stained with DAPI (blue). Scale bar: $100 \mu \mathrm{m}$. b Quantification of MUC5B staining. c Quantification of KRT5 staining. $\mathbf{d}$ Immunoblot analysis of lysates of bronchospheres cultured in the presence of flagellin, LPS, or control media probed with antibody to MUC5B or $\beta$-actin. e Immunohistochemistry was performed for MUC5AC. Scale bar: $20 \mu$ m. $\mathbf{f}$ Quantification of MUC5AC staining. Data were compared by unpaired Student's t-test and are shown as the mean \pm SEM from three independent experiments. ${ }^{*} p<0.05,{ }^{* *} p<0.01$

lipopeptides). In the present study, we show by using an in vitro organoid model for the differentiation of mucociliary epithelium from basal cells [10] that exposure to flagellin results in mucus hyperproduction and loss of ciliation, whereas exposure to the synthetic lipopeptide Pam3CSK only increased mucus production. These results suggest that prolonged activation of innate immune mechanisms by bacteria via PRRs such as TLR- 5 drives 
the differentiation of basal cells towards mucusproducing goblet cells and suppresses formation of cilia. Thus, the interaction of bacterial components with respiratory cells may promote basal and goblet cell metaplasia and hinder mucociliary clearance as well as the regeneration of injured epithelium and tissue homeostasis in the airways of CF and COPD patients.

$P$. aeruginosa-derived flagellin had a strong effect on the differentiation of the mucociliary epithelium. Studies showed that HBECs cells detect $P$. aeruginosa via recognition of flagellin by TLR- 5 and that approximately $75 \%$ of clinical isolates of $P$. aeruginosa retain TLR5 activating capacity during chronic CF lung infection [14, 15]. However, as cytosolic receptors (e.g. NLRC4 inflammasome receptors) also detect flagellin [16] we cannot exclude signaling pathways independent of TLR-5 contributing to the flagellin-induced mucus hyperproduction. In any case, our data indicate that bacterial components can cause structural changes in the airways of CF patients independent of CFTR (Cystic Fibrosis Transmembrane Conductance Regulator). This assumption is support by findings of Adam et al. showing that air liquid interface cultures (ALI) with freshly isolated cells obtained from CF patients have a hyperinflammatory phenotype and exhibit basal cell hyperplasia without increased numbers of goblet cells as compared to cultures with cells obtained from non-CF patients. However, ALI cultures with passages $1 \mathrm{CF}$ cells did not show a hyperinflammatory phenotype anymore, nor did they longer reconstitute a remodeled epithelium. Moreover, a mixture of pro-inflammatory cytokines increased numbers of goblet cells independent of mutations in CFTR [17]. Thus, goblet metaplasia and mucus hyperproduction may relate to bacterial infections and inflammation rather than to CFTR dysfunction $[1,17]$.

Danahay et al. showed that incubation of developing bronchospheres with interleukin (IL)-13 and IL-17A, a cytokine that is suggested to mediate $P$. aeruginosa-induced lung inflammation $[18,19]$, results in increased mucin production and decreased expression of markers of ciliated cells in an Notch2-dependent manner [10]. Our data indicate that direct activation of basal cells by bacteria also promotes goblet cell metaplasia and airway remodeling in chronic respiratory diseases. Additional studies are needed to clarify whether there are specific signaling pathways that regulate cell differentiation in response to cytokines and bacteria and whether cytokines and bacteria synergistically induce goblet cell metaplasia.

In summary, our data indicate that ongoing activation of innate immune pathways in basal cells promotes goblet cell metaplasia and airway remodeling as seen in chronic respiratory diseases such as CF and COPD. Thus, 3D bronchosphere models are useful tools for the identification of druggable signaling pathways that mediate mucus hyperproduction and loss of ciliation, thereby reducing and replacing animal studies. As therapeutic interventions targeting basal cell differentiation and mucus hyperproduction have the potential to improve lung function in CF and COPD one approach could be the inhibition of TLR, inflammasome, or Notch signaling pathways using small molecules or antibodies in 3D bronchospheres models $[15,20]$.

\begin{abstract}
Abbreviations
CF: Cystic fibrosis; CFTR: Cystic fibrosis transmembrane conductance regulator; COPD: Chronic obstructive pulmonary disease; IPF: Idiopathic pulmonary fibrosis; PAMPs: Pathogen-associated molecular patterns; TLR: Toll-like receptor; plC: Polyinosinic:polycytidylic acid; HBECs: Human bronchial epithelial cells; NTHi: Nontypeable Haemophilus influenzae; Muc5B: Mucin 5B; KRT5: Cytokeratin 5; ALl: Air liquid interface
\end{abstract}

\section{Acknowledgements}

We thank Andreas Kamyschnikow and Anja Honecker (Department of Internal Medicine V, Saarland University) as well Alexander Grissmer and Belinda König for excellent technical assistance and Carola Meier (Institute of Anatomy and Cell Biology, Saarland University) for support with the analysis of ultrastructure.

\section{Authors' contributions}

R.F.S., F.R., C.B: designed the study, involved in conducting experiments, analyzed data, wrote the manuscript. C.H., Y.Y., F.L.: involved in conducting experiments, wrote the manuscript. Y.K., T.T.; R.B.: designed the study, analyzed data, wrote the manuscript. The author (s) read and approved the final manuscript.

\section{Funding}

This study was supported by grants from Stiftung "Forschung für Leben" and Stiftung SET.

\section{Availability of data and materials}

The datasets generated and analyzed during the current study are available from the corresponding author on reasonable request.

\section{Ethics approval and consent to participate}

The protocol for isolation of primary cells was approved by the institutional review board (ethics committee) of the Landesärztekammer of the State of Saarland, and informed consent was obtained from the patients.

\section{Competing interests}

None of the authors have a financial relationship with a commercial entity that has an interest in the subject of this manuscript. Robert Bals is the Editor-in-Chief of Respiratory Research starting from Feb 1st 2020.

\section{Author details}

${ }^{1}$ Department of Internal Medicine V - Pulmonology, Allergology and Respiratory Critical Care Medicine, Saarland University, Kirrberger Str. 100, Building 41M, 66421 Homburg/Saar, Germany. ${ }^{2}$ Department of Thoracic and Cardiovascular Surgery, Saarland University, 66421 Homburg, Germany. ${ }^{3}$ Department Bioprocessing \& Bioanalytics, Fraunhofer Institute for Biomedical Engineering, Joseph-von-Fraunhofer-Weg 1, 66280 Sulzbach, Germany. ${ }^{4}$ Institute of Anatomy and Cell Biology, Saarland University, 66421 Homburg, Germany.

Received: 31 January 2020 Accepted: 13 August 2020

Published online: 26 August 2020

\section{References}

1. De Rose V, Molloy K, Gohy S, Pilette C, Greene CM. Airway epithelium dysfunction in cystic fibrosis and COPD. Mediat Inflamm. 2018;2018:1309746.

2. Shaykhiev R. Emerging biology of persistent mucous cell hyperplasia in COPD. Thorax. 2019;74:4-6. 
3. Ralhan A, Laval J, Lelis F, Ballbach M, Grund C, Hector A, Hartl D. Current concepts and controversies in innate immunity of cystic fibrosis lung disease. J Innate Immun. 2016;8:531-40.

4. Sethi S. Infection as a comorbidity of COPD. Eur Respir J. 2010;35:1209-15.

5. Hartl D, Gaggar A, Bruscia E, Hector A, Marcos V, Jung A, Greene C, McElvaney G, Mall M, Doring G. Innate immunity in cystic fibrosis lung disease. J Cyst Fibros. 2012;11:363-82.

6. Hartl D, Tirouvanziam R, Laval J, Greene CM, Habiel D, Sharma L, Yildirim AO, Dela Cruz CS, Hogaboam CM. Innate immunity of the lung: from basic mechanisms to translational medicine. J Innate Immun. 2018;10:487-501.

7. Dinh PC, Cores J, Hensley MT, Vandergriff AC, Tang J, Allen TA, Caranasos TG, Adler KB, Lobo LJ, Cheng K. Derivation of therapeutic lung spheroid cells from minimally invasive transbronchial pulmonary biopsies. Respir Res. 2017;18:132

8. Henry E, Cores J, Hensley MT, Anthony S, Vandergriff A, de Andrade JB, Allen T, Caranasos TG, Lobo L, Cheng K: Adult lung spheroid cells contain progenitor cells and mediate regeneration in rodents with bleomycininduced pulmonary fibrosis. Stem Cells Transl Med 2015, 4:1265-1274.

9. Barkauskas CE, Chung MI, Fioret B, Gao X, Katsura H, Hogan BL. Lung organoids: current uses and future promise. Development. 2017;144:986-97.

10. Danahay H, Pessotti AD, Coote J, Montgomery BE, Xia D, Wilson A, Yang H, Wang Z, Bevan L, Thomas C, et al. Notch2 is required for inflammatory cytokine-driven goblet cell metaplasia in the lung. Cell Rep. 2015;10:239-52.

11. Bals R, Beisswenger C, Blouquit S, Chinet T. Isolation and air-liquid interface culture of human large airway and bronchiolar epithelial cells. J Cyst Fibros. 2004;3(Suppl 2):49-51.

12. Ritzmann F, Jungnickel C, Vella G, Kamyschnikow A, Herr C, Li D, Menger MM, Angenendt A, Hoth M, Lis A, et al. IL-17C-mediated innate inflammation decreases the response to PD-1 blockade in a model of Krasdriven lung cancer. Sci Rep. 2019;9:10353.

13. Schmiedl A, Kerber-Momot T, Munder A, Pabst R, Tschernig T. Bacterial distribution in lung parenchyma early after pulmonary infection with Pseudomonas aeruginosa. Cell Tissue Res. 2010;342:67-73.

14. Zhang Z, Louboutin JP, Weiner DJ, Goldberg JB, Wilson JM. Human airway epithelial cells sense Pseudomonas aeruginosa infection via recognition of flagellin by toll-like receptor 5. Infect Immun. 2005;73:7151-60.

15. Blohmke CJ, Victor RE, Hirschfeld AF, Elias IM, Hancock DG, Lane CR, Davidson AG, Wilcox PG, Smith KD, Overhage J, et al. Innate immunity mediated by TLR5 as a novel antiinflammatory target for cystic fibrosis lung disease. J Immunol. 2008;180:7764-73.

16. Zhao Y, Yang J, Shi J, Gong YN, Lu Q, Xu H, Liu L, Shao F. The NLRC4 inflammasome receptors for bacterial flagellin and type III secretion apparatus. Nature. 2011;477:596-600.

17. Adam D, Roux-Delrieu J, Luczka E, Bonnomet A, Lesage J, Merol JC, Polette M, Abely M, Coraux C. Cystic fibrosis airway epithelium remodelling: involvement of inflammation. J Pathol. 2015;235:408-19.

18. Wonnenberg B, Jungnickel $C$, Honecker A, Wolf L, Voss M, Bischoff M, Tschernig T, Herr C, Bals R, Beisswenger C. IL-17A attracts inflammatory cells in murine lung infection with P. aeruginosa. Innate Immun. 2016;22:620-5.

19. Muir R, Osbourn M, Dubois AV, Doran E, Small DM, Monahan A, O'Kane CM, McAllister K, Fitzgerald DC, Kissenpfennig A, et al. Innate lymphoid cells are the predominant source of IL-17A during the early pathogenesis of acute respiratory distress syndrome. Am J Respir Crit Care Med. 2016;193:407-16.

20. Wu Y, Cain-Hom C, Choy L, Hagenbeek TJ, de Leon GP, Chen Y, Finkle D, Venook R, Wu X, Ridgway J, et al. Therapeutic antibody targeting of individual notch receptors. Nature. 2010;464:1052-7.

\section{Publisher's Note}

Springer Nature remains neutral with regard to jurisdictional claims in published maps and institutional affiliations.

Ready to submit your research? Choose BMC and benefit from:

- fast, convenient online submission

- thorough peer review by experienced researchers in your field

- rapid publication on acceptance

- support for research data, including large and complex data types

- gold Open Access which fosters wider collaboration and increased citations

- maximum visibility for your research: over $100 \mathrm{M}$ website views per year

At BMC, research is always in progress.

Learn more biomedcentral.com/submissions 